\title{
ОПИСАНИЕ НЕСТАЦИОНАРНОГО РАСПРЕДЕЛЕНИЯ НАПОРА В МНОГОСЛОЙНОЙ ВОДОВМЕЩАЮЩЕЙ ТОЛЩЕ С ПОМОЩЬЮ СИСТЕМЫ ДИФФЕРЕНЦИАЛЬНЫХ УРАВНЕНИИ
}

Для исследования нестационарной фильтрации в многослойной водовмещающей толще, где вода перетекает через разделяющие слои, может быть применена система дифференциальных уравнений (Гавич, 1983; Шестаков, 1979; Hantush, 1960)

$$
\begin{aligned}
& \frac{\partial}{\partial x}\left(T_{i} \frac{\partial H_{i}}{\partial x}\right)+\frac{\partial}{\partial y}\left(T_{i} \frac{\partial H_{i}}{\partial y}\right)+k_{i-1} \frac{\partial \bar{H}_{i-1}}{\partial z}\left(t, x, y, z_{i}\right)- \\
& -k_{i} \frac{\partial \bar{H}_{i}}{\partial z}\left(t, x, y, z_{i}\right)+Q_{i}=S_{i} \frac{\partial H_{i}}{\partial t} \\
& v_{i} \frac{\partial^{2} \bar{H}_{i}}{\partial z^{2}}=\frac{\partial \bar{H}_{i}}{\partial t}
\end{aligned}
$$

Здесь $T_{i}(x, y)$ - водопроводимость $i$-го водоносного горизонта $(i=1, \ldots, n) ; H_{i}(t, x, y)$ - напор в водоносном горизонте; $k_{i-1} ; k_{i}-$ коэффициенты фильтрации разделяющих слоев; $Q_{i}(t, x, y)$ - откачка или нагнетание воды через скважины; $S_{i}(x, y)$ - упругая водоотдача водоносного горизонта; $\bar{H}_{i}(t, z)$ и $v_{i}$ - соответственно напор и пьезопроводность разделяющего слоя. При этом предполагается, что нестационарная фильтрация в слоях относительно высокой водопроводимости (в водоносных горизонтах) происходит на плоскостях $x, y, z_{i}$, а в разделяющих водоносные горизонты относительно слабо проводящих слоях - только в направлении вертикальной оси $z$ (рис. $1 a, \sigma)$.

Таким образом, комплекс водоносных горизонтов, из которых каждый имеет определенную мощность $b_{i}$, заменяется, согласно системе (1) - (2), параллельными плоскостями $x, y, z_{i}$ (рис. 1 б), причем соблюдается условие

$$
H_{i}(t, x, y)=\bar{H}_{i-1}\left(t, x, y, z_{i}\right)=\bar{H}_{i}\left(t, x, y, z_{i}\right) .
$$

Решения системы дифференциальных уравнений (1)-(2) рассмотрены уже ранее (Herrera, 1970, 1974; Herrera, Figueroa, 1969; Herrera, Rodarte, 1973; Herrera, Yates, 1977; Rodarte, 1976, 1978) при начальном условии

для трех случаев:

$$
H_{i}(0, x, y)=\bar{H}_{i}(0, z)=0
$$

$$
\begin{gathered}
\bar{H}_{0}(t, x, y, 0)=\bar{H}_{n}\left(t, x, y, z_{n+1}\right)=0 ; \\
\frac{\partial \bar{H}_{0}}{\partial z}(t, x, y, 0)=\frac{\partial \bar{H}_{n}}{\partial z}\left(t, x, y, z_{n+1}\right)=0 ; \\
\bar{H}_{0}(t, x, y, 0)=0 ; \quad \frac{\partial \bar{H}_{n}}{\partial z}\left(t, x, y, z_{n+1}\right)=0 .
\end{gathered}
$$


Граничных условий уравнений, заданных на плоскостях $x, y, z_{i}$, при этом не устанавливали. Физическая сущность условий (3)-(7) следующая: рассматриваетсл водовмещающая толща, верхний и нижний слои которой слабопроницаемы: описывается развитие понижения напора, обусловленное откачкой воды через скважины из водоносных горизонтов. В первом случае (5) вся изучаемая толща ограничена сверху и снизу поверхностями $x, y, 0$ и $x, y, z_{n+1}$, на которых напор остается все время постоянным; во втором случае (6) водовмещающая толща находится между двумя абсолютно водонепроницаемыми поверхностями. В третьем случае (7) толща залегает на абсолютно водонепроницаемой поверхности $x, y, z_{n+1}$, а на кровле толщи $x, y, 0$ всегда должен сохраняться постоянный напор.

Условия (5) - (6) с гидрогеологической точки зрения трудно выполнимы или же имеют весьма ограниченное распространение. Например, строго говоря, постоянный напор может существовать на верхней поверхности эксплуатируемой водовмещающей толщи только тогда, когда на толще залегает поверхностный водоем (водохранилище), в котором искусственно поддерживается неизменный уровень воды. Сохранять постоянный напор в подстилающем эксплуатируемую водовмещающую толщу слое практически невозможно. Правда, предпосылка о постоянном напоре в ограничивающихся слоях может быть принята условно, имея в виду, что напор там изменяется не так существенно, как в подвергающейся откачке толще, но такое допущение понижает точность результатов расчета. Предпосылка об абсолютной изолированности водовмещающей толщи как сверху, так и снизу обоснована лишь в начальной стадии откачки или тогда, когда суммарное количество откачиваемой воды незначительно.

Более реально условие (7). Оно подходит для изучения распространения понижения в эксплуатируемой водовмещающей толще, которая снизу изолирована, при предположении, что во время откачки над поверхностью $x, y, 0$ сохраняется некоторая средняя величина напора.

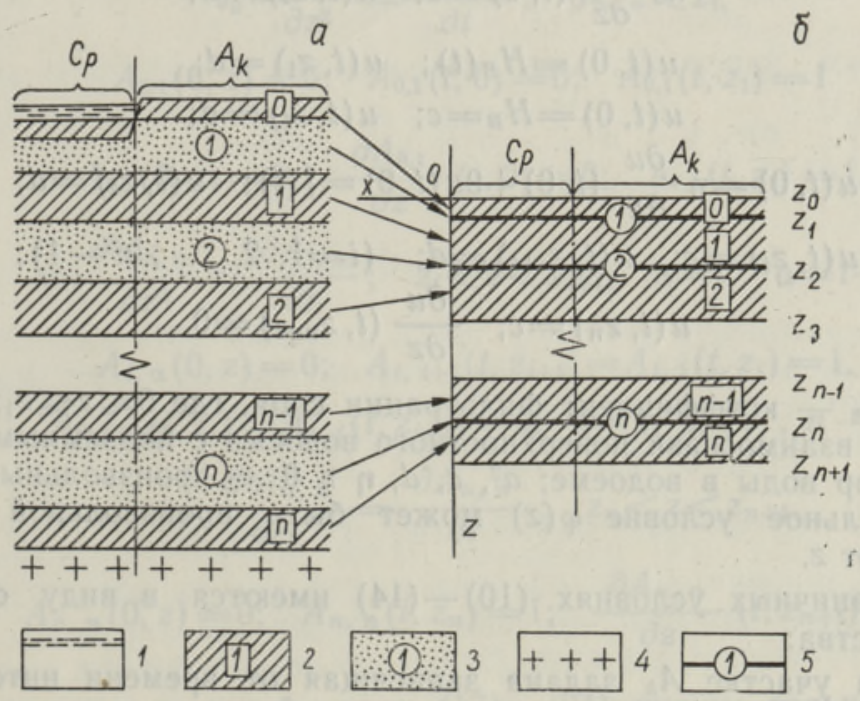

Рис. 1. Фильтрационная схема в разрезе. $a$ - многослойная водовмещающая толща, 6 - математическая модель водовмещающей толщи. В подобласти $A_{h}$ распространяется зона аэрации, в подобласти $C_{p}-$ поверхностный водоем. $I$ - поверхностный водоем, 2 - относительно слабопроницаемый разделяющий слой, 3 - водоносный горизонт, 4 - кристаллический фундамент, 5 - моделирующая водоносный горизонт поверхность $x, y, z_{i}$. 
Эта предпосылка выполняется тем лучше, чем меньше дебит откачки.

Ниже постараемся видоизменить модель И. Геррера таким образом, чтобы она лучше подходила для изучения реальных проблем геофильтрации. Рассмотрим многослойную водовмещающую толщу, которая в плане занимает некоторую замкнутую область $L$ (рис. 2 ). Предположим, что верхний слой толщи, где фильтрация происходит по вертикальной оси $z$, подразделяется на непересекающиеся участки $A_{k}$, $B_{m}, C_{p}, D_{r}$ и $E_{s}$, причем $k=1,2, \ldots, v_{1} ; m=1,2, \ldots, v_{2} ; p=1,2, \ldots, v_{3}$; $r=1,2, \ldots, v_{4} ; s=1,2, \ldots, v_{5}$. Снизу водовмещающая толща ограничена слабопроницаемым слоем, нижняя поверхность которого $\left(x, y, z_{n+1}\right)$ абсолютно водонепроницаема (этому соответствует залегание водовмещающей толщи, например, на кристаллическом фундаменте).

Будем считать, что на участках $A_{k}$ и $B_{m}$ верхний слой толщи представляет собой зону аэрации над грунтовым водоносным горизонтом. Ее верхняя граница $x, y, 0$ отождествляема с поверхностью земли, а нижняя $x, y, z_{1}-$ со средней высотой свободной поверхности грунтовых вод. Участки $C_{p}, D_{r}$ и $E_{s}$ занимают поверхностные водоемы различного типа, которые подстилаются в интервале $0 \leqslant z \leqslant z_{1}$ относительно слабопроницаемым слоем.

Для изучения фильтрации в рассматриваемой толще вводим вспомогательную функцию $u(t, x, y, z)$, удовлетворяющую уравнению

$$
a^{2} \frac{\partial^{2} u}{\partial z^{2}}=\frac{\partial u}{\partial t}
$$

при начальном условии

$$
u(0, z)=\varphi(z)
$$

и при следующих сочетаниях граничных условий:

$$
\begin{gathered}
k_{0} \frac{\partial u}{\partial z}(t, 0)=q_{0}(t, 0) ; \quad u\left(t, z_{1}\right)=d ; \\
k_{0} \frac{\partial u}{\partial z}(t, 0)=c ; \quad u\left(t, z_{1}\right)=d ; \\
u(t, 0)=H_{B}(t) ; \quad u\left(t, z_{1}\right)=d ; \\
u(t, 0)=H_{B}=c ; \quad u\left(t, z_{1}\right)=d ; \\
u(t, 0)=\eta \frac{\partial u}{\partial x}(t, 0)+\theta u(t, 0)=f(t) ; u\left(t, z_{1}\right)=d ; \\
u\left(t, z_{i}\right)=c ; \quad u\left(t, z_{i+1}\right)=d ; \quad(i=1,2, \ldots ; n-1) ; \\
u\left(t, z_{n}\right)=c ; \quad \frac{\partial u}{\partial z}\left(t, z_{n+1}\right)=0 .
\end{gathered}
$$

Здесь $k_{0}-$ коэффициент фильтрации слоя, где $0 \leqslant z \leqslant z_{1} ; \eta$ и $\theta-$ параметры взаимосвязи поверхностного водоема с подземными водами; $H_{B}$ - напор воды в водоеме; $a^{2}, c, d, \eta$ и $\theta-$ произвольные постоянные. Начальное условие $\varphi(z)$ может быть произвольной заданной функцией от $z$.

При граничных условиях (10)-(14) имеются в виду следующие обстоятельства:

(10) - на участке $A_{k}$ задана зависящая от времени интенсивность фактического питания грунтовых вод сверху;

(11) - на участке $B_{m}$ принято фактическое питание грунтовых вод, равное постоянной величине (например, приравнено к среднему многолетнему значению естественных ресурсов грунтовых вод);

(12) - на участке $C_{p}$ расположено море, большое озеро или река, на уровень воды в которых откачка из водовмещающей толщи не оказы- 


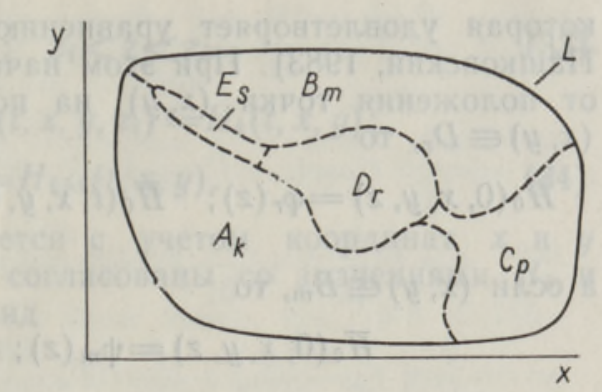

Рнс. 2. Фильтрационная схема в плане. Штриховой линией обозначены границы подобластей, по которым задаются условия согласования.

вает влияния; ввиду этого граничное условие на поверхности $x, y, 0$ определяется только колебанием уровня воды $H_{B}(t)$ в водоеме;

(13) - участок $D_{r}$ занимает поверхностный водоем, где уровень воды либо поддерживается искусственно на постоянной высоте (водохранилище), либо колеблется несущественно по оравнению с изменением напора в подвергающейся откачке водовмещающей толще;

(14) - на участке $E_{s}$ распространяется водоем (например, река), причем расход воды через ложе водоема на поверхности $x, y, 0$ зависит как от уровня воды в водоеме, так и от напора в слое, где $0 \geqslant z \geqslant z_{1}$; задано граничное условие третьего рода.

Решения уравнения (8) при условиях (9)-(16) хорошо известны, они выражаются через бесконечные ряды (Карслоу, Егер, 1964). Тем не менее, задачи (8)-(16), по-видимому, целесообразнее решать подходящими численными методами (Марчук, 1980). Ниже будем считать, что решения этих задач нам известны. Обозначим их соответственно через $u^{(\varepsilon)}(t, z)$, причем верхний индекс $\varepsilon=10,11, \ldots, 16$ означает номер рассмотренных выше граничных условий.

Далее необходимы (Herrera, 1970) вспомогательные функции $A_{0,1}, A_{i, i}$, $A_{i, i+1}(i=1,2, \ldots, n-1)$, удовлетворяющие уравнениям:

или

$$
v_{0} \frac{\partial^{2} A_{0,1}}{\partial z^{2}}=\frac{\partial A_{0,1}}{\partial t} ; 0<z<z_{1}
$$

$$
A_{0,1}(0, z)=0 ; \quad A_{0,1}(t, 0)=0 ; \quad A_{0,1}\left(t, z_{1}\right)=1
$$

$$
\begin{gathered}
A_{0,1}(0, z)=0 ; \quad \frac{\partial A_{0,1}}{\partial z}(t, 0)=0 ; \quad A_{0,1}\left(t, z_{1}\right)=1, \\
v_{i} \frac{\partial^{2} A_{i, \alpha}}{\partial z^{2}}=\frac{\partial A_{i, \alpha}}{\partial t} ; \quad z_{i}<z<z_{i+1} ; \quad \alpha=i ; \quad \alpha=i+1, \\
A_{i, \alpha}(0, z)=0 ; \quad A_{i, i+1}\left(t, z_{i+1}\right)=A_{i, i}\left(t, z_{i}\right)=1, \\
A_{i, i+1}\left(t, z_{i}\right)=A_{i, i}\left(t, z_{i+1}\right)=0 ; \quad i=1,2, \ldots, n-1, \\
v_{n} \frac{\partial^{2} A_{n, n}}{\partial z^{2}}=\frac{\partial A_{n, n}}{\partial t} ; \quad z_{n}<z<z_{n+1}, \\
A_{n, n}(0, z)=0 ; \quad A_{n, n}\left(t, z_{n}\right)=1 ; \quad \frac{\partial A_{n, n}}{\partial z}\left(t, z_{n+1}\right)=0 .
\end{gathered}
$$

Явные виды решений задач (17)-(24) нужны только при выводе окончательных уравнений для определения функций $H_{i}(t, x, y, z)$. В самих уравнениях решения задач (17)-(24) не фигурируют и поэтому мы их здесь не приводим.

Опишем теперь вывод уравнений для напора в первом случае, где $0<z<z_{1}$ и в водоносном горизонте, где $z=z_{1}$. Согласно принятым обозначениям, напор в слое $0<z<z_{1}$, описывается функцией $\bar{H}_{0}(t, x, y, z)$, 
которая удовлетворяет уравнению (2) (Бадов, Киселев, 1982, 1983 : Пашковский, 1983). При этом начальные и граничные условия зависят от положения точки $(x, y)$ на поверхности $x, y, 0$. Если, например, $(x, y) \in D_{r}$, то

$$
\bar{H}_{0}(0, x, y, z)=\varphi_{r}(z) ; \quad \bar{H}_{0}(t, x, y, 0)=c_{r} ; \quad \bar{H}_{0}\left(t, x, y, z_{1}\right)=H_{1}(t, x, y),
$$

а если $(x, y) \in B_{m}$, то

$$
\begin{gathered}
\bar{H}_{0}(0, x, y, z)=\psi_{m}(z) ; \quad k_{0} \frac{\partial \bar{H}_{0}}{\partial z}(t, x, y, 0)=c_{m} \\
\bar{H}_{0}\left(t, x, y, z_{1}\right)=H_{1}(t, x, y) .
\end{gathered}
$$

Начальное распределение напора в слое $\boldsymbol{z}=z_{1}$ задаем также кусочнопостоянным, т. е. $H_{1}(0, x, y)=\varphi_{r}\left(z_{1}\right)=\alpha_{r}$, если $(x, y) \in D_{r}$ и $H_{1}(0, x, y)=$ $=\psi_{m}\left(z_{1}\right)=\gamma_{m}$, если $(x, y) \in B_{m}$. Таким же образом задаются начальные условия и в последующих слоях, где $z=z_{i}$.

Воспользуясь решениями вспомогательного уравнения (8) и (17), можно записать:

$$
\bar{H}_{0}(t, x, y, z)=\int_{0}^{t} \frac{\partial H_{1}}{\partial \tau}(\tau, x, y) A_{0,1}(t-\tau, z) d \tau+u^{(13)}(t, z),
$$

если $(x, y) \in D_{r}$. Здесь $u^{(13)}(t, z)$ решение уравнения (8) при условиях (9) и (13), если $\varphi(z)=\varphi_{r}(z) ; c=c_{r} ; d=\alpha_{r}$ и $a^{2}=v_{0}$, а $A_{0,1}(t, z)$ является решением уравнения (17) при условиях (18).

Если $(x, y) \in B_{m}$, то

$$
\bar{H}_{0}(t, x, y, z)=\int_{0}^{t} \frac{\partial H_{1}}{\partial \tau}(\tau, x, y) A_{0,1}(t-\tau, z) d \tau+u^{(11)}(t, z)
$$

где $u^{(11)}(t, z)$ - решение уравнения (8) при условиях (9) и (11), где $\varphi(z)=\psi_{m}(z) ; c=c_{m} ; d=\gamma_{m}$ и $a^{2}=v_{0} . A_{0,1}$ - решение (17) при условиях (19). 1960)

В слое $z=z_{1}$, напор $H_{1}(t, x, y)$ удовлетворяет уравнению (Hantush,

$$
\begin{gathered}
\frac{\partial}{\partial x}\left(T_{1} \frac{\partial H_{1}}{\partial x}\right)+\frac{\partial}{\partial y}\left(T_{1} \frac{\partial H_{1}}{\partial y}\right)+k_{0} \frac{\partial \bar{H}_{0}}{\partial z}\left(t, x, y, z_{1}\right)- \\
-k_{1} \frac{\partial \bar{H}_{1}}{\partial z}\left(t, x, y, z_{1}\right)+Q_{1}=S_{1} \frac{\partial H_{1}}{\partial t} .
\end{gathered}
$$

Здесь, в зависимости от $x$ и $y$, соотношениями (27) или (28) определяется $\bar{H}_{0}$, а к определению $\bar{H}_{1}(t, x, y, z)$ придем ниже. Уравнение (29) решается при начальных условиях

$$
\begin{array}{cc}
H_{1}(0, x, y)=\varphi_{r}\left(z_{1}\right)=\alpha_{r} ; & (x, y) \in D_{r} ; \\
H_{1}(0, x, y)=\varphi_{m}\left(z_{1}\right)=\gamma_{m} ; & (x, y) \in B_{m} .
\end{array}
$$

Граничные условия и вид области $L$, где задача рассматривается, задаются отдельно для каждого конкретного случая.

Выпишем уравнение для слоя $z=z_{i}(i=2,3, \ldots, n-1)$

$$
\begin{gathered}
\frac{\partial}{\partial x}\left(T_{i} \frac{\partial H_{i}}{\partial x}\right)+\frac{\partial}{\partial y}\left(T_{i} \frac{\partial H_{i}}{\partial y}\right)+k_{i-1} \frac{\partial \bar{H}_{i-1}}{\partial z}\left(t, x, y, z_{i}\right)- \\
-k_{i} \frac{\partial \bar{H}_{i}}{\partial z}\left(t, x, y, z_{i}\right)+Q_{i}=S_{i} \frac{\partial H_{i}}{\partial t}
\end{gathered}
$$

Здесь $\bar{H}_{i}\left(t, x, y, z_{i}\right)$ удовлетворяет уравнению 


$$
\begin{gathered}
v_{i} \frac{\partial^{2} \vec{H}_{i}}{\partial z^{2}}=\frac{\partial \bar{H}_{i}}{\partial t} ; \quad z_{i}<z<z_{i+1} \\
H_{i}(0, x, y, z)=\varphi(z) ; \quad \bar{H}_{i}\left(t, x, y, z_{i}\right)=H_{i}(t, x, y) \\
\bar{H}_{i}\left(t, x, y, z_{i+1}\right)=H_{i+1}(t, x, y) .
\end{gathered}
$$

Начальное условие $\varphi(z)$ задается с учетом координат $x$ и $y$ функциями $\varphi_{r}(z)$ и $\psi_{m}(z)$, которые согласованы со значениями $H_{i}$ и $H_{i+1}$ при $t=0$. Тогда решение имеет вид

$$
\begin{aligned}
& \bar{H}_{i}(t, x, y, z)=\int_{0}^{t} \frac{\partial H_{i}}{\partial \tau}(\tau, x, y) A_{i, i}(t-\tau, z) d \tau+ \\
& +\int_{0}^{t} \frac{\partial H_{i+1}}{\partial \tau}(\tau, x, y) A_{i, i+1}(t-\tau, z) d \tau+u^{(15)}(t, z) .
\end{aligned}
$$

Здесь $u^{(15)}(t, z)$ - решение уравнения (8) при условиях (9) и (15), которые зависят также от положения точки $(x, y)$

$$
\begin{gathered}
u^{(15)}(0, z)=\varphi_{r}(z) \quad\left(\text { или }=\psi_{m}(z)\right) \\
u^{(15)}\left(t, z_{i}\right)=c=H_{i}(0, x, y) ; \quad u^{(15)}\left(t, z_{i+1}\right)=d=H_{i+1}(0, x, y) .
\end{gathered}
$$

В нижнем слое $z=z_{n}$ и

$$
\begin{gathered}
\frac{\partial}{\partial x}\left(T_{n} \frac{\partial H_{n}}{\partial x}\right)+\frac{\partial}{\partial y}\left(T_{n} \frac{\partial H_{n}}{\partial y}\right)+k_{n-1} \frac{\partial \bar{H}_{n-1}}{\partial z}\left(t, x, y, z_{n}\right)- \\
-k_{n} \frac{\partial \bar{H}_{n}}{\partial z}\left(t, x, y, z_{n}\right)+Q_{n}=S_{n} \frac{\partial H_{n}}{\partial t} .
\end{gathered}
$$

Аналогично предыдущему,

$$
v_{n} \frac{\partial^{2} \bar{H}_{n}}{\partial z^{2}}=\frac{\partial \bar{H}_{n}}{\partial t} ; \quad z_{n}<z<z_{n+1}
$$

$\bar{H}_{n}(0, x, y, z)=\varphi(z) ; \quad \bar{H}_{n}\left(t, x, y, z_{n}\right)=H_{n}(t, x, y) ; \quad \frac{\partial \bar{H}_{n}}{\partial z}\left(t, x, y, z_{n+1}\right)=0$ и

$$
\bar{H}_{n}(t, x, y, z)=\int_{0}^{t} \frac{\partial H_{n}}{\partial z}(\tau, x, y) A_{n, n}(t-\tau, z) d \tau+u^{(16)}(t, z) .
$$

Здесь $u^{(16)}(t, z)$ удовлетворяет уравнению (8) при условиях (9) и (16), где $a^{2}=v_{n}$, начальное условие $\varphi(z)$ задается в зависимости от положения точки $(x, y)$ функциями $\varphi_{r}(z)$ или $\psi_{m}(z) ; u^{(16)}(t, z)=c=$ $=H_{n}(0, x, y)$.

Как показали И. Геррера, Л. Родарте и другие, а в последствии и Дж. Премчитт (Premchitt, 1981), уравнения (29), (32) и (36) можно упростить, заменив их приближенными. В итоге получим следующую систему уравнений для общего случая, при котором в области $L$ могут встречаться все подобласти $A_{k}, B_{m}, C_{p}, D_{r}$ и $E_{s}$ :

$$
\begin{gathered}
\frac{\partial}{\partial x}\left(T_{1} \frac{\partial H_{1}}{\partial x}\right)+\frac{\partial}{\partial y}\left(T_{1} \frac{\partial H_{1}}{\partial y}\right)+\frac{k_{1}}{\beta_{1}} H_{2}\left(t-t_{1,2}^{\star}\right)+k_{0} \frac{\partial u_{1}^{(\varepsilon)}}{\partial z}\left(t, z_{1}\right)- \\
-k_{1} \frac{\partial u_{1}^{(15)}}{\partial z}\left(t, z_{1}\right)-c_{1} H_{1}(t)+Q_{1}=\left(S_{1}+I_{1}\right) \frac{\partial H_{1}}{\partial t}
\end{gathered}
$$




$$
\begin{gathered}
\quad \frac{\partial}{\partial x}\left(T_{i} \frac{\partial H_{i}}{\partial x}\right)+\frac{\partial}{\partial y}\left(T_{i} \frac{\partial H_{i}}{\partial y}\right)+\frac{k_{i-1}}{\beta_{i-1}} H_{i-1}\left(t-t_{i, i-1}^{*}\right)+ \\
+\frac{k_{i}}{\beta_{i}} H_{i+1}\left(t-t_{i, i+1}^{*}\right)+k_{i-1} \frac{\partial u_{i-1}^{(15)}}{\partial z}\left(t, z_{i}\right)-k_{i} \frac{\partial u_{i}^{(15)}}{\partial z}\left(t, z_{i}\right)- \\
\quad-\left(\frac{k_{i-1}}{\beta_{i-1}}+\frac{k_{i}}{\beta_{i}}\right) H_{i}(t)+Q_{i}=\left[S_{i}+\frac{1}{3}\left(\bar{S}_{i-1}+\bar{S}_{i}\right)\right] \frac{\partial H_{i}}{\partial t} \\
\frac{\partial}{\partial x}\left(T_{n} \frac{\partial H_{n}}{\partial x}\right)+\frac{\partial}{\partial y}\left(T_{n} \frac{\partial H_{n}}{\partial y}\right)+\frac{k_{n-1}}{\beta_{n-1}} H_{n-1}\left(t-t_{n, n-1}^{*}\right)+ \\
+k_{n-1} \frac{\partial u_{n-1}^{(15)}}{\partial z}\left(t, z_{n}\right)-k_{n} \frac{\partial u_{n}^{(16)}}{\partial z}\left(t, z_{n}\right)-\frac{k_{n-1}}{\beta_{n-1}} H_{n}(t)+Q= \\
=\left[S_{n}+\frac{1}{3} \bar{S}_{n-1}+\bar{S}_{n}\right] \frac{\partial H_{n}}{\partial t} .
\end{gathered}
$$

Здесь $t_{i, i+1}^{*}=\beta_{i}^{2} / 6 v_{i}(i=1, \ldots, n-1) ; t_{i, i-1}^{*}=\beta_{i-1}^{2} / 6 v_{i-1}(i=2, \ldots$ $\ldots, n) ; \beta_{1}$ и $\bar{S}_{i}$ - соответспвенно мощность и удельная упругоемкость разделяющего слоя. В областях $C_{p}$ и $D_{r}$ величина $I_{1}=$ $=\left(\bar{S}_{0}+\bar{S}_{1}\right) / 3$ и $c_{1}=k_{0} / \beta_{0}+k_{1} / \beta_{1}$, а в областях $A_{k}$ и $B_{m}: \quad I_{1}=\bar{S}_{0}+\bar{S}_{1} / 3 ;$ $c_{1}=k_{1} / \beta_{1}$. Нижний индекс функции $u$ обозначает номер слоя, причем верхний индекс $\varepsilon$ в уравнении (39) может приобрести значения $10, \ldots, 14$, в зависимости от характера граничных условий на поверхности $x, y, 0$.

В начале вычислений все уравнения системы (39)-(41) решаются отдельно. Если, например, В $i$-м уравнении $t<t_{i, i-1}^{*}$ н $t<t_{i, i+1}^{*}$, то соответственно, $H_{i-1}\left(t-t_{i, i-1}^{*}\right) \equiv 0$, и $H_{i+1}\left(t-t_{i, i+1}^{*}\right) \equiv 0$. Начиная с моментов $t=t_{i, i-1}^{*}$ и $t=t_{i, i+1}^{*}$ появляются члены $H_{i-1}\left(t-t^{*}{ }_{i, i+1}\right)$ и $H_{i+1}\left(t-t_{i, i+1}^{*}\right)$, которые учитываются в соседних слоях. Чтобы решить уравнения (39)-(41) во всей рассматриваемой области $L$, нужно задать условия согласования (условия сопряженности) на границах соседних подобластей $A_{k}, B_{m}, C_{p}, D_{r}$ и $E_{s}$. Одно из необходимых условий -. равенство значений функций $H_{i}(t, x, y)$ на этих границах, другое равенство производных функций $H_{i}(t, x, y)$ на границах подобластей.

Итак, благодаря содержанию в системе (39)-(41) функции $u_{i}^{(\varepsilon)}(t, z)$ представляется возможным учесть практически все виды

краевых условий, существующих на верхней границе водовмещающей толщи с любым количеством чередующихся водоносных горизонтов и разделяющих их слабопроницаемых слоев. На основании модификаций решений системы уравнений (39)-(41), кроме нестационарного распределения напора в общем случае, могут быть изучены распространение различных возмущений напора (например, понижения, влияния колебания напора в водоемах и т.д.), баланс подземного стока, а также оседание земной поверхности, обусловленное эксплуатацией подземных вод.

ЛИТЕРАТУРА

Бадов В. В., Киселев А. А. Совместное движение грунтовых вод и влаги в зоне аэрацин. - Вод. ресурсы, 1982, 1, 16-26.

Бадов В. В., Киселев А. А. Численное решение задачи взаимодействия поверхностных и подземных вод. - Вод. ресурсы, 1983, 1, 66-80. 
Гавич Н. K. Теоретйческие основы изучения движения̆ подземных водд в земной коре. - В кн.: Основы гидрогеологии. Гидрогеодинамика. М., 1983, 7-105. Карслоу Г., Ееер Д. Теплопроводность твердых тел. М., 1964.

Марчук Г. Н. Методы вычислительной математики. М., 1980.

Пашковский И. С. Закономерности процессов насыщения и осушения водоносных горизонтов. - В кн.: Гидрогеология и инженерная геология. Обзорная информация ВИЭМС. М., 1983.

Шестаков В. М. Динамика подземных вод. М., 1979.

Hantush, M. S. Modification of the theory of leaky aquifers. - J. Geophys. Res., 1960, 65, 3713-3725.

Herrera, I. Theory of multiple leaky aquifers. - Water Resour. Res., 1970, 6, 185193.

Herrera, I. Integrodifferential equations for systems of leaky aquifers and applications, 2. Error analysis of approximate theories. - Water Resour. Res., 1974, 10, $811-820$.

Herrera, I., Figueroa, G. E. A correspondence principle for the theory of leaky aquifers. - Water Resour. Res., 1969, 5, 900-904.

Herrera, I., Rodarte, L. Integrodifferential equations for systems of leaky aquifers and applications. 1. The nature of approximate theories. - Water Resour. Res., 1973, 9, 995-1005.

Herrera, I., Yates, R. Integrodifferential equations for systems of leaky aquifers and applications. 3. A numerical method of unlimited applicability. - Water Resour. Res., 1977, 13, 725-732.

Rodarte, $L$. Theory of multiple leaky aquifers. 1. The integrodifferential and differential equations for small and large values of time. - Water Resour. Res., 1976, 12, $163-170$.

Rodarte, $L$. An approximate differential equation to describe leaky aquifer behavior during intermediate and large values of time. - Water Resour. Res., 1978, 14, 39-44.

Premchitt, J. A technique in using integrodifferential equations for model simulation of multiaquifer systems. - Water Resour. Res., 1981, 17, 162-168.

\section{Ннститут геологии \\ Академии наук Эстонской ССР \\ Институт кибернетики \\ Академии наук Эстонской ССР}

Поступила в редакцию 30/I 1984

\section{VALLNER, T. TOBIAS}

\section{DIFERENTSIAALVORRANDITE SUSTEEM MITTESTATSIONAARSE ROHU JAOTUSE KIRJELDAMISEKS PALJUKIHILISES POHJAVEELADESTUS}

Mittestatsionaarse kvaasi-kolmemõõtmelise rõhu jaotus paljukihilises heterogeenses põhjaveeladestus on kirjeldatav diferentsiaalvõrrandite süsteemiga (1) - (2) rajatingimustel (9) - (16). Seda süsteemi võib lähendada võrrandisüsteemiga (39) - (41), mis sobib numbriliseks lahendamiseks.

\section{VALLNER, T. TOBIAS}

\section{DIFFERENTIAL EQUATIONS TO DESCRIBE THE PIESOMETRIC HEAD IN A SYSTEM OF LEAKY AQUIFERS}

Under the assumption of horizontal flow in the $n$ elastic inhomogeneous aquifers $(n \geqslant 2)$ and vertical flow in the aquitards, the mathematical description of the nonsteady piesometric head $H$ is given by the system of partial differential equations (1)-(2) (Fig. 1). The initial head is given by an arbitrary function of coordinates $x, y, z_{i}$. In the domain $L$ (Fig. 2) leaky aquifers are covered either by the aeration zone (subdomains $A_{k}, B_{m}$ ) or by bottom sediments of a stream, lake or sea (subdomains $\left.C_{p}, D_{r}, E_{s}\right)$. On their upper surface where $z=0$, the boundary conditions are $(10)-(15)$. On horizontal limits of aquifers the boundary conditions are not specified. On the surface $z=z_{n+1}$ the system of aquifers is limited by an impervious layer. The equations (1) - (2) governing the head $H$ in cases of practical interest given by conditions (9) $-(16)$ are transferred to a simpler system (39)-(41) suitable for numerical solution. 